Byung Lae Park $\cdot$ Hyun Sub Cheong · Lyoung Hyo Kim

Yoo Hyun Choi · Sohg Namgoong · Hae-Sim Park

Soo-Jong Hong · Byoung Whui Choi

June Hyuk Lee $\cdot$ Choon-Sik Park $\cdot$ Hyoung Doo Shin

\title{
Association analysis of signal transducer and activator of transcription 4 (STAT4) polymorphisms with asthma
}

Received: 10 November 2004 / Accepted: 24 December 2004/Published online: 3 March 2005

(C) The Japan Society of Human Genetics and Springer-Verlag 2005

\begin{abstract}
The signal transducer and activator of transcription 4 (STAT4) on chromosome 2q32.2-q32.3 is known to be essential for mediating responses to interleukin 12 in lymphocytes and regulating the differentiation of $\mathrm{T}$ helper cells. In an effort to discover additional polymorphism(s) in genes in which variant(s) have been implicated in asthma, we investigated the genetic polymorphisms in STAT4 to evaluate it as a potential candidate gene for a host genetic study of asthma. By direct DNA sequencing in 24 individuals, we identified 12 sequence variants within introns and their flanking regions, including the $1.5 \mathrm{~kb}$ promoter region of STAT4. Among them, seven common polymorphic sites were selected for genotyping in our asthma cohort (502 asthmatic patients, 164 normal controls). Using logistic regression analysis for association with the risk of asthma, while controlling for age, gender, and smoking status as covariates, no significant associations with the risk of asthma were detected. However, one single nucleotide polymorphism (SNP) in intron 11
\end{abstract}

B. L. Park · H. S. Cheong · L. H. Kim · Y. H. Choi

S. Namgoong $\cdot$ H. D. Shin

Department of Genetic Epidemiology, SNP Genetics, Inc.,

11th Floor, MaeHun B/D, 13 Chongro $4 \mathrm{Ga}$,

Chongro-Gu, Seoul, Korea, 110-834

H.-S. Park · S.-J. Hong · B. W. Choi · J. H. Lee · C.-S. Park Asthma Genome Research Group,

Soonchunhyang University Hospital,

Ajuo University Hospital,

Ulsan University Hospital,

and Choong-Ang University Hospital, Korea

C.-S. Park $(\square)$

Division of Allergy and Respiratory Medicine,

Department of Internal Medicine,

Soonchunhyang University Bucheon Hospital,

1174, Jung Dong, Wonmi Ku, Bucheon,

Gyeonggi Do, Korea, 420-021

E-mail: mdcspark@unitel.co.kr

Tel.: + 82-32-621-5105

Fax: $+82-32-621-5023$
$(+90089 T \rightarrow C)$ and two haplotypes showed positive association $(P=0.03,0.03$ and 0.03 , respectively) with production of specific IgE to Dermatophagoides farinae (D.f.) or Dermatophagoides pteronyssinus (D.p.) among asthmatic patients. The minor allele frequencies of $+90089 T \rightarrow C$ and BLOCK2-ht 1 were higher (0.54 and 0.47 , respectively) among individuals who produced specific $\operatorname{IgE}$ to D.f. or D.p. than frequencies $(0.47$ and 0.39 , respectively) among individuals who did not produce specific $\operatorname{IgE}(\mathrm{OR}=1.38$ and 1.40 , respectively). Our findings suggest that polymorphisms in STAT4 might be one of the genetic factors for the risk of production of specific IgE to mite allergens.

Keywords Asthma STAT4 $\cdot$ Single nucleotide polymorphism $\cdot$ Specific IgE

\section{Introduction}

Asthma and its phenotypes are complex traits induced by interactions between multiple genetic susceptible factors and the surrounding environment (Sengler et al. 2002). With advances in the understanding of molecular and cellular mechanisms involved in the asthmatic response, researchers have identified several specific mediators that may be targeted to control asthma.

The signal transducer and activator of transcription 4 (STAT4) on chromosome 2q32.2-q32.3 is known to be essential for mediating responses to interleukin 12 (IL12) in lymphocytes and for regulating the differentiation of $\mathrm{T}$ helper cells. This region has been linked to atopy susceptibility in previous genome-wide scans (Koppelman et al. 2002). IL12 is an important immunoregulatory cytokine whose receptor is a member of the hematoprotein receptor super family, implicating these kinases in the immediate biochemical responses to IL12 (Bacon et al. 1995). Previous studies demonstrated that 
IL12 induced tyrosine phosphorylation of signal transducers and activators of transcription (STAT) family members and that STAT4 expression was regulated by T-cell activation (Cho et al. 1996). The activated STAT4 is an essential element in the early events of Th1 differentiation through its effects on IL12 signaling (Nishikomori et al. 2002).

The $\mathrm{T}$ helper type 2 (Th2) hypothesis (Lordan et al. 2002) proposes that the inflammation in asthma arises from an imbalance between the two CD4 + T lymphocyte subsets: T helper type 1 (Th1) and Th2. The Th1 cytokine, IL12, has been a target of intense study because it mediates the Th1 response and induces the asthmatic inflammatory response (Birkisson et al. 2004). STATs are a family of latent cytoplasmic transcription factors. Upon cytokine stimulation, STATs become tyrosine phosphorylated and then form homo- or heterodimers that translocate to the cell nucleus where they act as transcription activators (Levy and Darnell 2002; O'Shea et al. 2002). Based on the biological properties involved in the essential role of mediating responses to IL12 in lymphocytes and regulating the differentiation of $\mathrm{T}$ helper cells as above, it is hypothesized that STAT4 plays an important role in asthma development.

We performed extensive screening of STAT4 by direct sequencing to detect polymorphisms and examined genetic association with the risk of asthma. Here, we present 12 genetic polymorphisms found in STAT4 and the results of an association study in a Korean asthma cohort.

\section{Materials and methods}

\section{Subjects}

Subjects were recruited from the Asthma Genome Research Center that consists of four tertiary hospitals in Korea (Soonchunhyang University Hospital, Ajuo University Hospital, Ulsan University Hospital, and Choong-Ang University Hospital). The Institutional Review Board of each of the hospitals approved the study, and informed consent was obtained from each and every subject prior to enrollment. Each patient presented with typical asthma symptoms, and physical signs were compatible with asthma with a reversible airway narrowing, as documented by an inhalant bronchodilator-induced improvement by more than $15 \%$ of forced expiratory volume in $1 \mathrm{~s}$ (FEV1) and/or an airway hyperreactivity to methacholine challenge of less than $10 \mathrm{mg} / \mathrm{ml}$. Control subjects consisted of the spouses of the patients as well as subjects randomly selected from the general population, all of whom answered negatively to a screening questionnaire for respiratory symptoms and had negative results of all laboratory tests, i.e., normal chest radiogram, FEV1 greater than $75 \%$ of predicted value, and methacholine concentration causing a $20 \%$ reduction in FEV1 $\left(\mathrm{PC}_{20}\right)$ greater than $10 \mathrm{mg} /$ $\mathrm{ml}$. Total IgE and specific IgE to Dermatophagoides farinae (D.f.) and D. pteronyssinus (D.p.) were also measured using the CAP system (Pharmacia Diagnostics, Sweden). Individuals who had scores of D.f. $\geq 1$ or D.p $\geq 1$ were regarded as positive for specific IgE, and all others were included in the negative group. Atopy was defined by the presence of an immediate skin reaction (greater than $3 \mathrm{~mm}$ in diameter) to one or more of 24 common aeroallergens, including dust mites (D.f. and D.p.), cat fur, dog fur, cockroaches, grass, tree pollens, and ragweed. The clinical parameters are summarized in Table 1.

Sequencing analysis of the STAT4 gene

We sequenced exons and their flanking regions, including the promoter region $(1.5 \mathrm{~kb})$ of $S T A T 4$, to discover variants in 24 Korean unregulated individual DNA samples using the ABI PRISM 3730 DNA analyzer (Applied Biosystems, Foster City, CA, USA). Twentyfive primer sets for the amplification and sequencing analysis were designed based on GenBank sequences (Ref. Genome seq.; NT_005403 released on 19 August 2004). Information regarding primers is available on our Web site (http://www.snp-genetics.com/user/additional_list.asp). Sequence variants were verified by chromatograms.

Genotyping by single-base extension and electrophoresis

For genotyping of polymorphic sites, amplifying and extension primers were designed for single-base extension (SBE) (Vreeland et al. 2002). Primer extension reactions were performed with the SNaPshot ddNTP Primer Extension Kit (Applied Biosystems). To clean up the primer extension reaction, one unit of SAP (shrimp alkaline phosphatase) was added to the reaction mixture, and the mixture was incubated at $37^{\circ} \mathrm{C}$ for $1 \mathrm{~h}$ followed by $15 \mathrm{~min}$ at $72^{\circ} \mathrm{C}$ for enzyme inactivation. The DNA samples, containing extension products, and

Table 1 Clinical profiles of the study subjects. FVC1 forced vital capacity in $1 \mathrm{~s}, F E V 1$ forced expiratory volume in $1 \mathrm{~s}, D . f$. Dermatophagoides farinae, D.p. Dermatophagoides pteronyssinus

\begin{tabular}{llc}
\hline Clinical profiles & $\begin{array}{l}\text { Normal } \\
\text { controls }\end{array}$ & Asthmatics \\
\hline Number of subjects & 164 & 502 \\
Age: mean (range) & $43.9(6-83)$ & $48.5(4-86)$ \\
Gender (male/female) & $74 / 90$ & $196 / 306$ \\
Current smoker & $30.66 \%$ & $34.10 \%$ \\
FVC1\%, predicted & $101.1 \pm 15.1$ & $80.2 \pm 22.7$ \\
FEV1\%, predicted & $92.4 \pm 12.0$ & $83.5 \pm 18.2$ \\
PC 20, methacholine (mg/ml) & $23.26 \pm 5.07$ & $6.21 \pm 8.55$ \\
Total IgE (IU/ml) & $143.9 \pm 284.2$ & $375.6 \pm 944.3$ \\
Blood eosinophil $(\%)$ & $2.58 \pm 2.12$ & $5.38 \pm 5.38$ \\
Positive rate of specific IgE (D.f.) & $24.32 \%$ & $35.08 \%$ \\
Positive rate of specific IgE (D.p.) & $31.86 \%$ & $43.22 \%$ \\
Positive rate of skin test & $34.15 \%$ & $57.09 \%$ \\
\end{tabular}


A Map of STAT4 on chromosome 2q32.2-q32.3

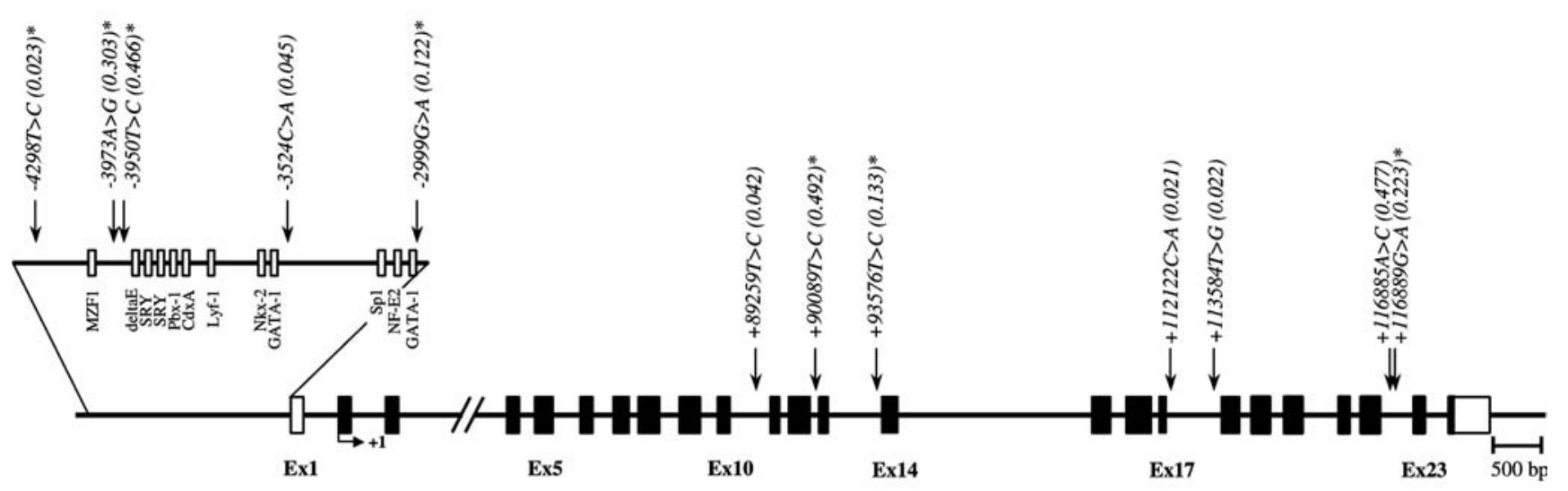

\section{B Haplotypes in STAT4}

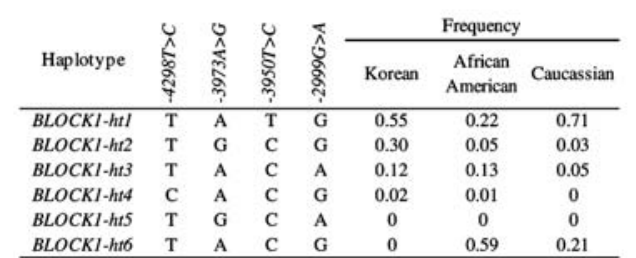

\begin{tabular}{|c|c|c|c|c|c|c|}
\hline \multirow[b]{2}{*}{ Haplotype } & \multirow{2}{*}{$\begin{array}{l}\text { U. } \\
\text { \&్ } \\
\text { \&్ } \\
+ \\
+\end{array}$} & \multirow{2}{*}{ 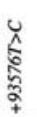 } & \multirow{2}{*}{ 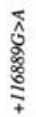 } & \multicolumn{3}{|c|}{ Frequency } \\
\hline & & & & Korean & $\begin{array}{c}\text { African } \\
\text { American }\end{array}$ & Caucassian \\
\hline BLOCK2-htI & $\mathrm{T}$ & $\mathrm{T}$ & G & 0.42 & 0.21 & 0.71 \\
\hline BLOCK2-ht2 & $\mathrm{C}$ & $\mathrm{T}$ & G & 0.34 & 0.65 & 0.14 \\
\hline BLOCK 2 -ht3 & $\mathrm{C}$ & $\mathrm{C}$ & A & 0.12 & 0.04 & 0.04 \\
\hline BLOCK2-ht4 & $\mathrm{T}$ & $\mathrm{T}$ & A & 0.08 & 0 & 0.10 \\
\hline BLOCK2-ht5 & C & $\mathrm{T}$ & A & 0.02 & 0 & 0 \\
\hline BLOCK2-ht6 & C & $\mathrm{C}$ & G & 0.02 & 0.10 & 0.01 \\
\hline
\end{tabular}

Fig. 1 Gene map, haplotypes, and linkage disequilibrium (LD) coefficients in STAT. a Gene map and single nucleotide polymorphisms (SNPs) in STAT4 on chromosome 2q32.2-q32.3. Coding exon is marked by black blocks and $5^{\prime}$ and 3' UTRs by white blocks. The first base of the translation site is denoted as nucleotide +1 . Putative transcription-factor-binding motifs are indicated (TFSEARCH Searching Transcription Factor Binding Sites V1.3. putative score $>0.9$ ). b Haplotypes in STAT4. c LD coefficient $\left(\left|\mathrm{D}^{\prime}\right|\right)$ and LD BLOCKs among STAT4 SNPs. Triangles indicate haplotype BLOCKs (denoted as BLOCK1 and BLOCK2)

Genescan 120-Liz-size standard solution were added to Hi-Di formamide (Applied Biosystems) according to the manufacturer's recommendation. The mixture was incubated at $95^{\circ} \mathrm{C}$ for $5 \mathrm{~min}$ followed by $5 \mathrm{~min}$ on ice, and then electrophoresis was performed using the ABI Prism 3100 Genetic Analyzer. The results were analyzed using the program of ABI Prism GeneScan and Genotyper (Applied Biosystems). Information regarding the primers is available on our Web site (http://www.snpgenetics.com/user/additional_list.asp).

\section{Statistics}

The $\chi^{2}$ tests were used to determine whether individual variants were in equilibrium at each locus in the popu-
C LDs among SNPs in STAT4

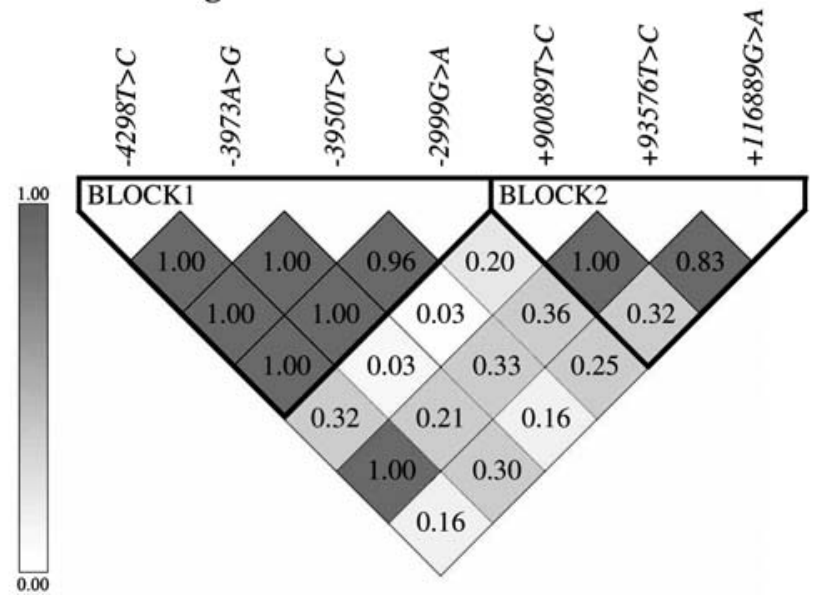

lation (Hardy-Weinberg equilibrium). We examined a widely used measure of linkage disequilibrium between all pairs of biallelic loci, Lewontin's $D^{\prime}\left(\left|D^{\prime}\right|\right)$ (Hedrick 1987). Haplotypes were inferred using the algorithm (Haploview) that searches for a spine of strong $\left|D^{\prime}\right|$ running from one marker to another (Barrett et al. 2004). Phase probabilities of each site were calculated for each individual by this software. Genotype distribution of STAT4 single nucleotide polymorphisms (SNPs) and haplotypes among asthmatics and normal subjects were analyzed with logistic regression models controlling for age (continuous value), gender (male = 0 , female $=1$ ), and smoking status (nonsmoker $=0$, exsmoker $=1$, smoker $=2$ ) as covariates, showing positive associations $(P=0.0006$ for age, $P=0.0009$ for gender, and $P<0.0001$ for smoking status, respectively) with the risk of asthma onset.

\section{Results}

Twelve SNPs (five in the $5^{\prime}$ flanking region and seven in introns) were identified by direct sequencing (Fig. 1a). Putative transcription factor-binding motifs on the $5^{\prime}$ upstream region were estimated (TFSEARCH Searching Transcription Factor Binding Sites V1.3. putative 
score $>0.9$ ), but five SNPs in $5^{\prime}$ upstream region did not affect transcription factor-binding motif. Seven SNPs were selected for larger-scale genotyping based on linkage disequilibriums (LDs) among SNPs and frequencies. Four polymorphisms $(-3524 C \rightarrow A,+890259 T \rightarrow C$, $+112122 C \rightarrow A$, and $+113584 T \rightarrow G$ ) showing low frequencies $(<0.05)$ were excluded for further analysis, and $+116885 A>C$, which was in almost absolute LD with $+90089 T \rightarrow C$, was also excluded. The minor allele frequencies of genotyped polymorphisms were 0.023 $(-4298 T \rightarrow C), \quad 0.303 \quad(-3973 A \rightarrow \quad G), \quad 0.466$ $(-3950 T \rightarrow C), \quad 0.122 \quad(-2999 G \rightarrow A), \quad 0.492$ $(+90089 T \rightarrow C), 0.133(+93576 T \rightarrow C)$, and 0.223 $(+116889 G \rightarrow A)$, respectively, in the Korean population $(n=666)$. LD coefficients $\left(\left|D^{\prime}\right|\right)$ and $r^{2}$ among polymorphisms were also calculated (Fig. 1). Interestingly, the analysis showed that STAT4 could be parsed into two haplotypes (BLOCK1 and 2; Fig. 1c), with each block having a strong LD spine. There were four and six haplotypes in BLOCK1 and BLOCK2, respectively. Significant differences in frequencies of SNPs and haplotypes were observed among three ethnic groups (Korean, African, and Caucasian populations) (Fig. 1c). Only common haplotypes (frequency $>0.05$ ) were used for further analysis. All haplotypes in BLOCK1 and BLOCK 2 - $h t 3$ were used for analysis because they were completely tagged by single SNPs (Fig. 1b).

Using logistic regression analysis for association with the risk of asthma controlling for age, gender, and smoking status as covariates, no significant associations with the risk of asthma (among all, atopic, and nonatopic subgroups) were detected (Table 2). However, one SNP in intron $12(+90089 T \rightarrow C)$ and two haplotypes in BLOCK2 (BLOCK2-ht1 and BLOCK2-ht2) showed positive association $(P=0.03,0.03$ and 0.03 , respectively) with plasma levels of specific IgE to D.f. or D.p. among asthmatic patients. The minor allele frequencies of $+90089 T \rightarrow C$ and $B L O C K 2-h t 1$ were higher $(0.54$ and 0.47 , respectively) among individuals who produced specific IgE to D.f. or D.p. (0.47 and 0.39 , respectively) than those among individuals who did not produce specific $\operatorname{IgE}(\mathrm{OR}=1.38$ and 1.40 , respectively). On the other hand, $B L O C K 2-h t 2$ had an opposite genetic effect on the specific $\operatorname{IgE}$ production $(P=0.03, \mathrm{OR}=0.71)$ (Table 3).

\section{Discussion}

The production of specific $\operatorname{IgE}$ is initiated by the activation of Th2 cells that release IL4 and IL13 in response to antigen presentation. It has been reported that mitespecific T-cell clones from donors with high levels of specific IgE produced significantly more IL4 and less IFN- $\gamma$ than clones from donors with low levels of specific IgE (Looney et al. 1994). In addition, IL4 production of $\mathrm{T}$ cells in response to house dust mites was shown to have a close correlation with serum-specific IgE (Koh et al. 2000). These results suggest that circulating allergen-specific $\mathrm{T}$ cells may be skewed toward a Th2 profile in patients with hypersensitivity to mites.

STAT4 is an essential element in the early events of Th1 differentiation (Nishikomori et al. 2002). Intraperitoneal injection of IL12, one of most important Th1 cytokines, inhibited the production of $\operatorname{IgE}$ and $\operatorname{IgG}$ antigen-specific antibodies on a murine model of asthma induced by the D.p. allergen in mice (Lee et al. 1999). IL12 has previously been shown to induce tyrosine phosphorylation and DNA-binding of STAT4 (STAT) family (Cho et al. 1996; O'Shea et al. 2002). In a previous study, the STAT4(-/-) mice showed significant decreases in airway hyperreactivity and peribronchial eosinophils compared with wild-type controls. In addition, pulmonary levels of chemokines were decreased in the STAT4(-/-) mice. These data suggest STAT4 expression contributes to allergen-induced chemokine

Table 2 Genotype distribution of STAT4 polymorphisms in bronchial asthmatics (BA) and normal controls (NC) in the Korean population

\begin{tabular}{|c|c|c|c|c|c|c|c|c|c|c|}
\hline \multirow[t]{3}{*}{ Locus } & \multirow[t]{3}{*}{ Position } & \multicolumn{3}{|l|}{ All subjects } & \multicolumn{3}{|l|}{ Atopic subjects } & \multicolumn{3}{|c|}{ Nonatopic subjects } \\
\hline & & \multicolumn{2}{|l|}{ Frequency $^{\mathrm{a}}$} & \multirow[t]{2}{*}{$P^{\mathrm{b}}$} & \multicolumn{2}{|l|}{ Frequency $^{\mathrm{a}}$} & \multirow[t]{2}{*}{$P^{\mathrm{b}}$} & \multicolumn{2}{|l|}{ Frequency $^{\mathrm{a}}$} & \multirow[t]{2}{*}{$P^{\mathrm{b}}$} \\
\hline & & $\mathrm{BA}(n=502)$ & $\mathrm{NC}(n=164)$ & & $\mathrm{BA}(n=364)$ & $\mathrm{NC}(n=72)$ & & $\mathrm{BA}(n=138)$ & $\mathrm{NC}(n=92)$ & \\
\hline$-1302 \mathrm{~T} \rightarrow C$ & Promoter & 0.02 & 0.02 & 0.66 & 0.03 & 0.03 & 0.96 & 0.02 & 0.01 & 0.66 \\
\hline$-977 \mathrm{~A} \rightarrow G$ & Promoter & 0.30 & 0.30 & 0.5 & 0.30 & 0.33 & 0.51 & 0.31 & 0.24 & 0.12 \\
\hline$-954 \mathrm{~T} \rightarrow C$ & Promoter & 0.45 & 0.44 & 0.39 & 0.45 & 0.47 & 0.72 & 0.43 & 0.37 & 0.20 \\
\hline$-3 \mathrm{G} \rightarrow A$ & Promoter & 0.12 & 0.12 & 0.97 & 0.13 & 0.11 & 0.71 & 0.09 & 0.13 & 0.65 \\
\hline$+93085 T \rightarrow C$ & Intron11 & 0.50 & 0.48 & 0.99 & 0.47 & 0.47 & 0.69 & 0.54 & 0.51 & 0.64 \\
\hline$+96572 T \rightarrow C$ & Intron 12 & 0.14 & 0.12 & 0.95 & 0.14 & 0.14 & 0.97 & 0.13 & 0.13 & 0.95 \\
\hline$+119885 G \rightarrow A$ & Intron21 & 0.22 & 0.23 & 0.74 & 0.22 & 0.20 & 0.63 & 0.22 & 0.25 & 0.25 \\
\hline Block2-ht 1 & - & 0.42 & 0.42 & 0.92 & 0.45 & 0.44 & 0.96 & 0.39 & 0.40 & 0.90 \\
\hline Block2-ht2 & - & 0.34 & 0.33 & 0.68 & 0.32 & 0.32 & 0.72 & 0.38 & 0.35 & 0.37 \\
\hline Block2-ht4 & - & 0.12 & 0.10 & 0.87 & 0.12 & 0.09 & 0.32 & 0.12 & 0.13 & 0.36 \\
\hline
\end{tabular}

\footnotetext{
${ }^{\text {a }}$ Minor allele frequencies

b Codominant $P$ values for logistic analyses controlling for smoking, age, and gender as covariates
} 
Table 3 Logistic analysis of STAT4 polymorphisms with the risk of specific IgE production [Dermatophagoides farinae (D.f.) or Dermatophagoides pteronyssinus (D.p)] among asthmatic subjects

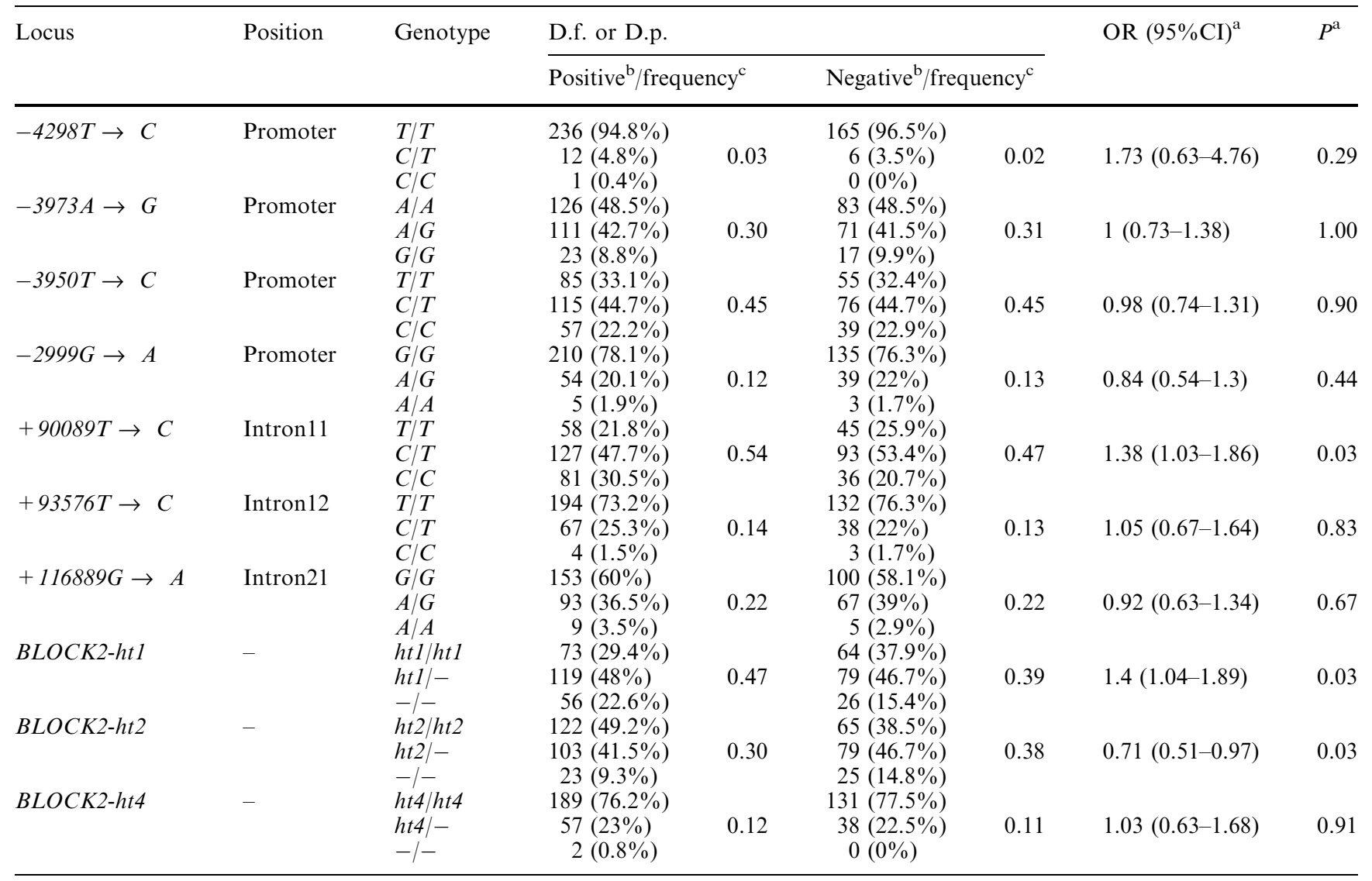

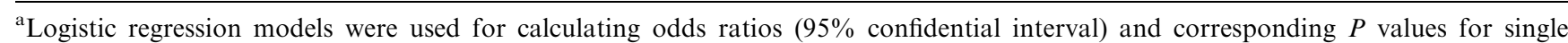
nucleotide polymorphism (SNP) sites, controlling for age, smoking, and gender as covariates

${ }^{b}$ Positive for specific IgE: individuals who had a score of D.f. $\geq 1$ or D.p. $\geq 1$. Other cases are in the negative group

${ }^{c}$ Rare allele frequency

production and airway hyperreactivity. Therefore, STAT4-mediated pathways play a role locally within the airway in the exacerbation of the allergen-induced responses (Raman et al. 2003).

In this study, polymorphisms in STAT4 (one intronic SNP and two haplotypes in BLOCK 2) showed positive association with the risk of production of specific IgE to mite allergens D.f. or D.p. among asthmatic patients. The LD among SNPs depends on a number of factors, including recombination, gene conversion, mutation, genetic drift, and selection (Twells et al. 2003). Haplotypes of STAT4 were divided into two parts: the $2 \mathrm{~kb}$ region of the promoter (BLOCK1) and gene region (BLOCK2) (Fig. 1b. Although five SNPs were identified on $5^{\prime}$ upstream region among 12 SNPs, these five SNPs did not affect regulatory motifs (Fig. 1a). Polymorphisms within introns can affect gene function by affecting both the splice donor or acceptor site or regions nearby and regulatory motifs within introns. However, $+90089 T \rightarrow C$ seemed to affect neither the alternative splicing event [NNSsplice version 0.9 (http://www.fruit- fly.org/seq_tools/splice.html)] nor the regulatory motifs (TFSEARC $\bar{C}$ ).

The effects of STAT4 polymorphisms on the production of specific $\operatorname{IgE}$ were not dramatic in the present study. When Bonferroni corrections were strictly adopted, associated $P$ values could not retain the significances (the threshold of significance would be 0.0025 (10 polymorphisms and two phenotypes analyzed). However, although there is a chance of type 1 error due to multiple comparisons, when considering the fact that the comparisons were not totally independent of each other due to tight LDs among SNPs/haplotypes, the significance of associations with specific IgE production among asthmatic patients might be noteworthy. Further biological and/or functional evidence would be needed to confirm the suggestive associations of STAT4 polymorphisms with asthma in this study.

In summary, we identified 12 sequence variants and have examined the association with risk of asthma and specific IgE production among asthmatic patients. Genetic analyses of STAT4 in asthmatic patients revealed 
putative associations of genetic polymorphisms with the risk of production of specific IgE to mite allergens.

Acknowledgements This work was supported by grant number M10302-00-0073 of the National Research Lab Program as part of the National Research and Development Program of the Ministry of Science and Technology of Korea.

\section{References}

Bacon CM, McVicar DW, Ortaldo JR, Rees RC, O'Shea JJ, Johnston JA (1995) Interleukin 12 (IL-12) induces tyrosine phosphorylation of JAK2 and TYK2: differential use of Janus family tyrosine kinases by IL-2 and IL-12. J Exp Med 181:399404

Barrett JC, Fry B, Maller J, Daly MJ (2005) Haploview: analysis and visualization of LD and haplotype maps. Bioinformatics 21(2):263-265

Birkisson IF, Halapi E, Bjornsdottir US, Shkolny DL, Adalsteinsdottir E, Arnason T, Gislason D, Gislason T, Gulcher J, Stefansson K, Hakonarson H (2004) Genetic approaches to assessing evidence for a $\mathrm{T}$ helper type 1 cytokine defect in adult asthma. Am J Respir Crit Care Med 169:1007-1013

Cho SS, Bacon CM, Sudarshan C, Rees RC, Finbloom D, Pine R, O'Shea JJ (1996) Activation of STAT4 by IL-12 and IFN-alpha: evidence for the involvement of ligand-induced tyrosine and serine phosphorylation. J Immunol 157:4781-4789

Hedrick PW (1987) Gametic disequilibrium measures: proceed with caution. Genetics 117:331-341

Koh YY, Jeong JH, Kim CK, Kim YK, Jee YK, Cho SH, Min KU, Kim YY (2000) Atopic status and level of bronchial responsiveness in parents of children with acute bronchiolitis. J Asthma 37:709-717

Koppelman GH, Stine OC, Xu J, Howard TD, Zheng SL, Kauffman HF, Bleecker ER, Meyers DA, Postma DS (2002) Genome-wide search for atopy susceptibility genes in Dutch families with asthma. J Allergy Clin Immunol 109:498-506
Lee YL, Fu CL, Ye YL, Chiang BL (1999) Administration of interleukin-12 prevents mite Der $p 1$ allergen-IgE antibody production and airway eosinophil infiltration in an animal model of airway inflammation. Scand J Immunol 49:229-236

Levy DE, Darnell JE, Jr (2002) Stats: transcriptional control and biological impact. Nat Rev Mol Cell Biol 3:651-662

Looney RJ, Pudiak D, Rosenfeld SI. (1994) Cytokine production by mite-specific $\mathrm{T}$ cells from donors with mild atopic disease. J Allergy Clin Immunol 93:476-483

Lordan JL, Bucchieri F, Richter A, Konstantinidis A, Holloway JW, Thornber M, Puddicombe SM, Buchanan D, Wilson SJ, Djukanovic R, Holgate ST Davies DE (2002) Cooperative effects of Th2 cytokines and allergen on normal and asthmatic bronchial epithelial cells. J Immunol 169:407-414

Nishikomori R, Usui T, Wu CY, Morinobu A, O'Shea JJ, Strober W (2002) Activated STAT4 has an essential role in Th1 differentiation and proliferation that is independent of its role in the maintenance of IL-12R beta 2 chain expression and signaling. J Immunol 169:4388-4398

O'Shea JJ, Gadina M, Schreiber RD (2002) Cytokine signaling in 2002: new surprises in the Jak/Stat pathway. Cell 109 (Suppl):S121-S131

Raman K, Kaplan MH, Hogaboam CM, Berlin A, Lukacs NW (2003) STAT4 signal pathways regulate inflammation and airway physiology changes in allergic airway inflammation locally via alteration of chemokines. J Immunol 170:3859-3865

Sengler C, Lau S, Wahn U, Nickel R (2002) Interactions between genes and environmental factors in asthma and atopy: new developments. Respir Res 3:7

Twells RC, Mein CA, Phillips MS, Hess JF, Veijola R, Gilbey M, Bright M, Metzker M, Lie BA, Kingsnorth A, Gregory E, Nakagawa Y, Snook H, Wang WY, Masters J, Johnson G, Eaves I, Howson JM, Clayton D, Cordell HJ, Nutland S, Rance H, Carr P, Todd JA (2003) Haplotype structure, LD blocks, and uneven recombination within the LRP5 gene. Genome Res 13:845-855

Vreeland WN, Meagher RJ, Barron AE (2002) Multiplexed, highthroughput genotyping by single-base extension and end-labeled free-solution electrophoresis. Anal Chem 74:4328-4333 